\title{
Another look at Linda
}

\author{
WAYNE S. MESSER and RICHARD A. GRIGGS \\ University of Florida, Gainesville, Florida
}

\begin{abstract}
Margolis (1987) argues that poor performance on the classic Linda conjunction problem is the result of misinterpretation created by multiple ambiguities in the problem statement acting in concert. He suggests three wording changes to rectify this situation: The addition of (1) a betting scenario to prevent the misinterpretation of "probable" as "believable" or "plausible," (2) a clarifying phrase to prevent misinterpretation of the response alternatives as mutually exclusive, and (3) survey information about the likelihood of personality profiles such as Linda's to ensure that subjects view the small difference between the extremely small probabilities to be judged as relevant. The effects of these three factors were examined in a between-subjects factorial design. Margolis's hypothesis was not supported. Only the addition of the response-alternative clarifying phrase significantly improved performance. The results are discussed in terms of Gigerenzer's (1991) normative-issues argument that the Linda problem is not a frequency probability problem but rather a single-trial, subjective probability problem.
\end{abstract}

In the present study, we take another look at the Linda problem, the classic conjunction problem in probability judgment research (Tversky \& Kahneman, 1982, 1983). In the Linda problem, subjects are provided with a summary description of a liberal-sounding individual before being asked to judge whether it is more probable that she is a bank teller or a feminist bank teller:

Linda is 31 years old, single, outspoken, and very bright. She majored in philosophy. As a student, she was deeply concerned with issues of discrimination and social justice, and also participated in anti-nuclear demonstrations.

Which of the following is more probable?

(Check one)

a) Linda is a bank teller.

b) Linda is a bank teller and is active in the feminist movement. (Tversky \& Kahneman, 1983, pp. 297, 299)

It is not unusual for $80 \%$ to $90 \%$ of the subjects, regardless of prior training in probabiliity and statistics, to choose the option of feminist bank teller (e.g., Tversky \& Kahneman, 1983; Wolford, Taylor, \& Beck, 1990). In some versions of the problem, subjects are asked to rank the probabilities of more alternatives, but the key comparison remains the same: The feminist bank teller option is judged to be more probable than the bank teller option.

Tversky and Kahneman (1983) have argued that the conjunction rule of probability theory is the normative arbiter for the Linda problem and dictates the bank teller option as the correct response (however, see Gigerenzer,

This article is based partially on a doctoral dissertation by the first author under the second author's supervision. The authors would like to thank Gerd Gigerenzer and Howard Margolis for valuable comments on an earlier version of this paper. Requests for reprints should be sent to W. S. Messer, who is now at the Department of Behavioral Sciences, Box 3471, High Point University, High Point, NC 27262, or to R. A. Griggs, Department of Psychology, University of Florida, Gainesville, FL 32611 .
1991). The conjunction rule states that the probability of two independent events occurring conjointly cannot be higher than the probability of either event occurring separately. Thus, it applies to cases of category membership in which one category subsumes the other. It is to this latter case of category membership that the Linda problem conforms.

Tversky and Kahneman (1983) have further argued that the major source of error is use of the representativeness heuristic. The representativeness heuristic is an abstract, content-independent strategy of judging similarity-the more similar, the more probable. In this case, the brief profile of Linda is judged to be similar to the stereotype of a feminist; thus, the joint event (because it contains the feminist category) is judged incorrectly to be more probable. Tversky and Kahneman have labeled this mistake the conjunction fallacy. In summary, they propose that subjects are incorrectly interpreting the problem intensionally in terms of plausibility via an intuitive heuristic, whereas they should interpret the problem extensionally in terms of set relations via the conjunction rule.

The present study comprises a test of Margolis's (1987) explanation of subjects' misinterpretation of the problem. Tversky and Kahneman (1983) and many others (e.g., Macdonald \& Gilhooly, 1990; Morier \& Borgida, 1984) have attempted to correct subjects' interpretations in a variety of ways. These researchers, however, have tended to focus on singular changes in the problem statement. Margolis's proposal is different in that he hypothesizes that there are multiple sources of ambiguity in the problem and that all of these need to be corrected in order to significantly impact performance. Curiously, the two major sources of misinterpretation that he proposes have been examined previously (even by Tversky \& Kahneman, 1983), but never in concert.

According to Margolis, poor performance on the Linda problem is the result of ambiguities at different levels. 
At one level, the subject does not even recognize the relevance of a set interpretation. At the other, the subject may consider a set interpretation but, in some way, still misrepresent the categories involved.

The individuating information about Linda (e.g., she is outspoken and bright) has no bearing on the probabilities to be judged. It is completely irrelevant information. Yet, it is given such salience as to create the expectation in the subject that such information is relevant to the judgment at hand. Given this framework, the subject may be led to interpret the problem as asking about the "believability" or "plausibility" that someone with a description such as Linda's could be a feminist. This aspect, however, is inherent in the problem and cannot be completely removed without also removing the very stereotypical aspect of the problem that is a test for use of the representativeness heuristic. Such problems will always be confounded in some degree by violation of the conversational principles of relevance. Margolis, however, has suggested changes to the problem that should lead people away from the assumption that the individuating information about Linda is supersedingly relevant without directly removing that information.

The tendency towards a believability or plausibility misinterpretation results from what Margolis calls scenario ambiguity, which, although inherent in the overall structure of the Linda problem, could possibly be ameliorated by the evocation of a "betting"' scenario. According to Margolis, the word probable, which Tversky and Kahneman chose for their instructions, is ambiguous. It can be interpreted in both the intended relative frequency and the nonintended believability assessment contexts. Instead of asking subjects to judge which alternative is "more probable," Margolis suggests that they be asked, "If you stood to win $\$ 10$ if the statement you choose turns out to be true, which choice is more likely to win you the \$10?' (Margolis, 1987, p. 166). There is some evidence that such a betting scenario may help. In accordance with similar reasoning, Tversky and Kahneman (1983) substituted a betting scenario for the typical probability judgment instruction that resulted in a $32 \%$ reduction in the conjunction error.

Margolis suggests that misinterpretations are also possible at the level of the response options. The two response options could be seen as being mutually exclusive. The response options could be read within a framework of "which is more probable, this option or this one?" Within conventional language usage, ors are often mutually exclusive, as in the question, "Are you having pie or pie a la mode?" With this interpretation, subjects are reading "Linda is a bank teller" as "Linda is a bank teller and is not active in the feminist movement." Given such a reading, the conjunction principle is no longer the normative arbiter for the problem; the judgment is between mutually exclusive events-"Linda is a bank teller and not active in the feminist movement" and "Linda is a bank teller and active in the feminist movement."
To make this misinterpretation less likely, Margolis proposes that a clarifying phrase ("whether or not the other statement is also true") be embedded in the betting scenario. Again, Tversky and Kahneman (1983) provide some evidence that the addition of a phrase to clarify the response alternatives may help subjects. They added a "whether or not she is also active in the feminist movement" phrase to Option a and reported a $25 \%$ reduction in the conjunction error as a result. In addition, Macdonald and Gilhooly (1990) also used a "who may or may not be active in the feminist movement" phrase, but only in concert with a problem version that projected judgments about Linda into the future, and found that it significantly lowered the conjunction error rate.

Margolis also posits a third, but more minor, difficulty with the problem. He points out that small differences between extremely small probabilities may not be pragmatically important. That is, the probabilities of Linda being a bank teller or a bank teller active in the feminist movement are so small that the misjudgment may be pragmatically unimportant. To combat this possible negligibility effect, Margolis proposed the addition of some survey information to the problem statement: "A personnel survey showed that of clerical workers in banks (including tellers), fewer than $1 \%$ have personality profiles that sound similar to Linda's"' (Margolis, 1987, p. 166). According to Margolis, this survey information should alert subjects to the fact that, in the case of either option, the probabilities are quite small but still relevant.

Margolis argues that subjects' difficulties with the Linda problem can be corrected if all three of his proposed changes are made. The present experiment was a test of that hypothesis.

\section{METHOD}

\section{Subjects}

Two hundred introductory psychology students at the University of Florida served as subjects in partial fulfillment of a research participation requirement. Twenty-five subjects were randomly assigned to each of eight conditions.

\section{Materials and Design}

The design was a $2 \times 2 \times 2$ between-subjects factorial design. The three factors were survey information (present vs. absent), scenario (betting vs. probability), and regardless phrase (present vs. absent). All eight problem versions used Tversky and Kahneman's description of Linda, which is given in the introduction.

The survey information factor consisted of either the absence or the presence of Margolis's proposed survey information. The survey information was inserted immediately after the description of Linda and read as follows: "A personnel survey showed that of clerical workers in banks (including tellers), fewer than $1 \%$ have personality profiles that sound similar to Linda's."

The betting scenario (or probability judgment instruction) followed the survey information in the survey-information-present problems but came directly after the Linda description in the survey-information-absent problems. Given their finding of facilitation for a betting scenario, Tversky and Kahneman's (1983) wording for the scenario was employed. It read as follows: "If you could win $\$ 10$ by betting on an event, which of the following would you choose to bet on? (Check one)." In the probabil- 
ity problems, the betting scenario was replaced with the typical judgment instruction: "Which of the following is more probable? (Check one)."

Each of the eight problems then offered two response options, $a$ and $b$. As in Tversky and Kahneman (1983), the regardless phrase factor was manipulated in Option a rather than in the betting scenario as recommended by Margolis. The placement of the clarification in the response option is a more direct way of combating the mutually exclusive misinterpretation.

In the regardless-phrase-absent problems, Option a read "Linda is a bank teller." In the regardless-phrase-present problems, Option a read "Linda is a bank teller, regardless of whether or not she is also active in the feminist movement." Option b always consisted solely of "Linda is a bank teller and is active in the feminist movement."

\section{Procedure}

The subjects were run in small groups, ranging in size from 5 to 15 . Each subject received one of the eight versions of the Linda problem; the order of administering the eight problems was randomized across groups.

\section{RESULTS}

The typical result for the standard Linda problem was replicated. There was an $88 \%$ error rate when the clarifying phrase, survey information, and betting scenario were not present. A three-factor analysis of variance revealed only a significant main effect for the regardless phrase factor $[F(1,192)=10.50, p<.05]$. The error rate was $21 \%$ lower for the regardless-present problems (56\% vs. $77 \%)$. Tversky and Kahneman's (1983) finding of facilitation for the betting scenario was not replicated ( $p=$ $.167) .{ }^{1}$ In fact, the error rate was greater when the betting scenario was present $(71 \%$ vs. $62 \%)$.

Although not significant at the .05 level, the two-way interactions involving the regardless phrase factor were marginally significant (both $p s<.10$ ). Multiple comparison follow-ups on these interactions revealed that the presence of either the survey information or the betting scenario reduced the effect of the clarifying phrase. Given the marginal significance of the interactions, no firm conclusions can be drawn from these analyses. It is, however, clear that the effects of the three factors are not additive as predicted by Margolis. A more complex interactive effect is indicated.

\section{DISCUSSION}

The results provide little support for Margolis's explanation of performance on the Linda problem. Admittedly, there are some minor differences between Margolis's proposed changes and those employed in the present study. These differences, however, stem mainly from using the wording of the betting scenario and the placement of the regardless phrase employed by Tversky and Kahneman (1983) that previously led to facilitation in both cases. Thus, these differences do not seem viable candidates for explaining the failure to observe much facilitation.

Tversky and Kahneman's (1983) finding of a $25 \%$ reduction in errors for the regardless phrase was replicated, but their finding of facilitation for the betting scenario was not. Given Macdonald and Gilhooly's (1990) finding of facilitation for two variants of the Linda problem that employed a similar regardless phrase and the failure of Wolford et al. (1990, Experiment 4) to find facilitation for two variants of the Linda problem employing a betting scenario, it would appear that the facilitation by the regardless phrase is robust (however, see Locksley \& Stangor, 1984 ) but that facilitation via the betting scenario is suspect. Nonetheless, the amount of facilitation for either factor on its own does not seem that great. The question arises as to why such manipulations have usually led to small effects, if any.

One possible answer emerges from a normative-issues argument by Gigerenzer (1991). He argues that extensional probability is not the correct normative arbiter in the Linda problem. He claims that subjects are asked to compare subjective probabilities of single events (i.e., that Linda is a bank teller and that Linda is a bank teller and active in the feminist movement) and that frequencies are not involved in the Linda problem as it is typically posed or even when the betting scenario or the response clarification phrase are added (also see Konold, 1989, for a discussion of a single-event interpretation of the Linda problem). Thus, the conjunction fallacy is not a violation of the major conception of probability, the frequentist view.

The lack of dramatic effects in the present study and most previous studies lends some support to Gigerenzer's argument. Stronger support, however, is furnished by Fiedler (1988). If Gigerenzer is right, then the conjunction fallacy should be drastically reduced if the problem is explicitly posed in a frequentist mode. Fiedler did this. He created frequency versions of the Linda problem by asking subjects to make frequency judgments instead of probability judgments. He asked subjects to provide numerical estimates for the different options when the total number of cases or events was always assumed to be 100 (Experiment 1) or for variable population sizes (Experiment 2). The effect of this manipulation was dramatic. Conjunction violation rates dropped to only $22 \%$ and $17 \%$ in the two experiments! ${ }^{2}$

Given the frequency-judgment results, what can be said about Margolis's proposal? While the specifics of Margolis's explanation appear to be wrong, his general idea of a scenario ambiguity might be modified to be congruent with the Gigerenzer argument and Fiedler's results. Like Gigerenzer, Margolis believes subjects can do frequency problems. Thus, Margolis could argue that the Linda problem cues the single-event scenario and the frequency-judgment problems cue the frequency scenario. Subjects make judgments in accordance with the specific scenario that is cued.

The implications of Gigerenzer's argument for the Tversky and Kahneman representativeness heuristic/conjunction fallacy approach are more severe. Simply put, if a "fallacy" is involved, it is probably more attributable to the researchers than to the subjects.

\section{REFERENCES}

FiedLeR, K. (1988). The dependence of the conjunction fallacy on subtle linguistic factors. Psychological Research, 50, 123-129.

GigerenZER, G. (1991). How to make cognitive illusions disappear: Beyond "heuristics and biases." In K. W. Stroebe \& M. Hewstone (Eds.), European review of social psychology (Volume 2, pp. 83-115). London: Wiley.

Konold, C. (1989). Informal conceptions of probability. Cognition \& Instruction, 6, 59-98.

Locksley, A., \& StANGor, C. (1984). Why versus how often: Causal reasoning and the incidence of judgmental bias. Journal of Experimental Social Psychology, 20, 470-483.

MACDONALD, R. R., \& Gilhooly, K. J. (1990). More about Linda or conjunctions in context. European Journal of Cognitive Psychology, 2, 57-70.

Margolis, H. (1987). Patterns, thinking, and cognition: A theory of judgment. Chicago: University of Chicago Press.

Morier, D. M., \& BorgiDA, E. (1984). The conjunction fallacy: A task specific phenomenon? Personality \& Social Psychology Bulletin, 10 , 243-252.

TVERSKy, A., \& KaHNEMAN, D. (1982). Judgments of and by representativeness. In D. Kahneman, P. Slovic, \& A. Tversky (Eds.), Judgment under uncertainty: Heuristics and biases (pp. 84-98). Cambridge: Cambridge University Press.

Tversky, A., \& KaHneman, D. (1983). Extensional versus intuitive reasoning: The conjunction fallacy in probability judgment. Psychological Review, 90, 293-315.

Wolford, G., TAYlor, H. A., \& Beck, J. R. (1990). The conjunction fallacy? Memory \& Cognition, 18, 47-53. 


\section{NOTES}

1. Both of these results (the significant effect of the regardless phrase factor and the lack of an effect of the betting factor) were replicated with two new groups of 20 subjects each from the same subject pool. The conjunction error rate for the Linda problem with just the regardless phrase added was only $65 \%$, but $90 \%$ with just the betting scenario added.
2. Curiously, Tversky and Kahneman (1983) also observed a dramatic drop in the error rate (only $25 \%$ and $11 \%$ ) for frequency rather than probability judgments in a health survey conjunction problem. Needless to say, their interpretation of these results was not concerned with normative issues.

(Manuscript received November 27, 1992.) 